\title{
Article \\ Combining Prognostic Nutritional Index and Brain Natriuretic Peptide as a Predicting Tool for Heart Transplantation
}

\author{
Ziwen Cai ${ }^{1,+}$, Jingrong $\mathrm{Tu}^{1,+}, \mathrm{Li} \mathrm{Xu}^{1}$, Yao Lin ${ }^{2}$, Bowen Deng ${ }^{3}$, Fei $\mathrm{Li}^{1,+}$, Si Chen ${ }^{1, * \mathbb{C}}$ and Nianguo Dong ${ }^{1, *}$ \\ 1 Department of Cardiovascular Surgery, Union Hospital, Tongji Medical College, Huazhong University of \\ Science and Technology, Wuhan 430022, China; caiziwen@hust.edu.cn (Z.C.); tirforwork@126.com (J.T.); \\ 2021507078@hust.edu.cn (L.X.); lifei_union@hust.edu.cn (F.L.) \\ 2 Department of Gastrointestinal Surgery, Union Hospital, Tongii Medical College, Huazhong University of \\ Science and Technology, Wuhan 430022, China; linyaomt@163.com \\ 3 The First Clinical College, Tongii Medical College, Huazhong University of Science and Technology, \\ Wuhan 430022, China; u201810271@hust.edu.cn \\ * Correspondence: Sichen@hust.edu.cn (S.C.); 1986xh0694@hust.edu.cn (N.D.) \\ $\dagger$ These authors contributed equally to this work.
}

check for updates

Citation: Cai, Z.; Tu, J.; Xu, L.; Lin, Y.; Deng, B.; Li, F.; Chen, S.; Dong, N. Combining Prognostic Nutritional Index and Brain Natriuretic Peptide as a Predicting Tool for Heart Transplantation. J. Cardiovasc. Dev. Dis. 2022, 9, 40. https://doi.org/ $10.3390 /$ jcdd 9020040

Academic Editors: Yasuhiro Shudo, Yoshiya Toyoda and Andy Wessels

Received: 15 November 2021

Accepted: 20 January 2022

Published: 24 January 2022

Publisher's Note: MDPI stays neutral with regard to jurisdictional claims in published maps and institutional affiliations.

Copyright: (C) 2022 by the authors. Licensee MDPI, Basel, Switzerland. This article is an open access article distributed under the terms and conditions of the Creative Commons Attribution (CC BY) license (https:// creativecommons.org/licenses/by/ $4.0 /)$.

\begin{abstract}
Our study aimed to evaluate the potential of prognostic nutritional index (PNI) and Brain natriuretic peptide (BNP) in predicting the prognosis of heart transplantation (HTx). We retrospectively investigated 489 patients undergoing HTx between 2015 and 2020 in our center. The relationship between preoperative index and prognosis was analyzed respectively, the optimal cut-off values for preoperative PNI and BNP level were evaluated with receiver operating curve analysis. Uni-variate analysis and multivariate analysis were used to compare baseline data (sex, age, diagnosis, etc.) of groups divided by the level of PNI and BNP. Propensity score matching (PSM) was applied to eliminate bias. We calculated the C-index from the prediction efficiency of PNI and BNP. During the period, 489 recipients undergoing HTx in our center were included according to the inclusion criteria; 383 (78.3\%) males and 106 (21.7\%) females were included in this study, with a median age of 47.57 years old. The ROC curve showed that the optimal cut-off values of each indicator were verified as 49.345 for PNI, and 4397.500 for BNP. The multivariate analyses indicated that PNI $(p=0.047)$, BNP $(p=0.024)$, age $(p=0.0023)$, and waiting time $(p=0.012)$ were risk factors for all-cause death after HTx. Propensity score matching generated 116 pairs based on PNI level and 126 pairs based on BNP level, and the results showed that OS (overall survival) was significantly correlated with PNI $(n=232, p=0.0113)$ and BNP $(n=252, p=0.0146)$. Our study implied that higher PNI and lower BNP level had direct correlation with better survival after HTx. Combining PNI and BNP together would be a potential clinical preoperative instrument to predict the survival of patients after HTx, especially in short-term survival.
\end{abstract}

Keywords: heart transplantation; prognostic nutritional index; brain natriuretic peptide; propensity score matching; China

\section{Introduction}

Heart transplantation (HTx) remains the "gold standard" procedure for patients with end-stage heart failure refractory to other treatments. The registry of International Society of Heart and Lung Transplantation (ISHLT) demonstrated the median survival in adult recipients between 2002 and 2009 was 12.5 years, and it increased to 14.8 years among 1-year survivors (ISHLT2019). The causes for short-term death includes acute graft failure, infectious complications, acute rejection, and renal failure. Moreover, the number of people on the waiting list for HTx far exceeds the number of people who will actually undergo HTx. Therefore, it is extremely necessary to identify the clinical and demographic characteristics of recipients, and find out the factors that could help to predict the prognosis of HTx. 
Heart-failure-related inflammation might bring about heart dysfunction including ventricular remodeling, cellular metabolic disorder, and cardiomyocyte necrosis. It is essential to investigate some valuable blood biomarkers that can be a tool for prognosis assessment after HTx. Brain natriuretic peptide (BNP) was recommended to be the biomarkers for diagnosis and prognosis of HF in the European clinical practice guidelines [1]. The prognostic nutritional index (PNI) reflects nutritional conditions of the human body and previous studies have demonstrated that PNI could be utilized as a useful indicator to predict prognosis in some cardiovascular diseases such as acute heart failure, coronary artery disease, and myocardial infarction [2-4]. However, no previous studies have focused on the relationship between PNI and the prognosis of HTx.

Accordingly, the aim of the present study was to investigate the prognostic values of BNP and PNI in HTx.

\section{Materials and Methods}

\subsection{Study Population}

All consecutive recipients of orthotopic heart transplantation $(n=581)$ were incorporated in this analysis at our center between 1 January 2015 and 31 December 2020. Multiple organ transplantation, re-transplantation, pediatric patients, and recipients with data missing were excluded $(n=92)$, and 489 patients were included in this study. Baseline demographic, clinical, and biochemical data for each patient were retrieved from electronic medical records. The Charlson Index based on a selected number of chronic diseases was used for general assessment of comorbidity status. Follow-up information was obtained for all survivors either through outpatient visit or by telephone interviews with the patients/their relatives, and was complete until 26 May 2021.

\subsection{Follow-Up Data and Variable Definitions}

Regular medical follow-up data were obtained using telephone calls, clinic visits, Internet, and other interaction tools. The all-cause overall survival (OS) rate was defined as the duration from transplantation surgery to the mortality event or the end of follow-up. Mortality data were obtained from China Heart Transplant Registration Network, where all deaths are registered, as required by law.

The demographic data included the sex, age, Body Mass Index (BMI), diagnosis, blood type, heart surgery history, Charlson Comorbidity Index, and waiting time on the list. The recipient/donor indicators included BMI, age, sex, and blood type. The pre-operative treatments included intra-aortic balloon pump (IABP), Cardiac Resynchronization Therapy Defibrillator (CRTD), extracorporeal membrane oxygenation (ECMO), ARB drug, ACEI drug, dopamine, and BB. The pre-operative laboratory indicators included white blood cell count (WBC), red blood cell count (RBC), and levels of blood platelet (PLT), hemoglobin $(\mathrm{Hb})$, glutamic oxaloacetic transaminase (AST), and alanine transaminase (ALT), D-dimer, creatinine $(\mathrm{Cr})$, troponin, brain natriuretic peptide (BNP), triglyceride (TG), and LDL. All pre-operative laboratory examinations were performed within 7 days before surgery. PNI was calculated as $10 \times$ serum albumin $(\mathrm{g} / \mathrm{dL})+0.005 \times 1$ ymphocyte count $\left(\right.$ per $\left.\mathrm{mm}^{3}\right)$.

\subsection{Statistical Analysis}

Categorical variables were presented as count of patients (percentage) and continuous variables as median (interquartile range (IQR)) or mean \pm standard deviation (SD), as appropriate. Descriptive comparisons were performed with Pearson's c2 for categorical variables and Mann-Whitney U rank sum test for continuous variables, as appropriate. Propensity score matching (PSM) was conducted on the basis of 19 clinically relevant variables. The quality of the matching was assessed by absolute standard differences, with a value $<5 \%$ considered as not significant. Univariate and multivariate Cox proportional hazard modeling was used to estimate the adjusted hazard ratio (HR) and 95\% confidence interval (CI) for clinical factors in OS. Survival analysis was generated with the KaplanMeier (KM) method, and differences between groups were examined with the log-rank 
test. For validating the discrimination of PNI and BNP in OS, we calculated the area under the ROC curve (AUC) in the cohort. Furthermore, the Harrell's C index was generated for discrimination of PNI and BNP using 1000 times bootstrap. Statistical significance was defined by a $p$ value of less than 0.05 (two-sided). Analysis was performed using $\mathrm{R}$ version 4.0.1 with the packages MatchIt and SPSS 26.0.

\section{Results}

Our study enrolled 489 patients according to the inclusion criteria (Figure 1 study flow chart); 383 (78.3\%) males and 106 (21.7\%) females were included in this study, with a median age of 47.57 years old. Among all patients, 95 patients were diagnosed as ischemic cardiomyopathy, 308 patients were diagnosed as non-ischemic cardiomyopathy, 19 were diagnosed as congenital heart disease, and 67 were diagnosed as other heart diseases (valvular cardiomyopathy and arrhythmic cardiomyopathy) (Table 1).

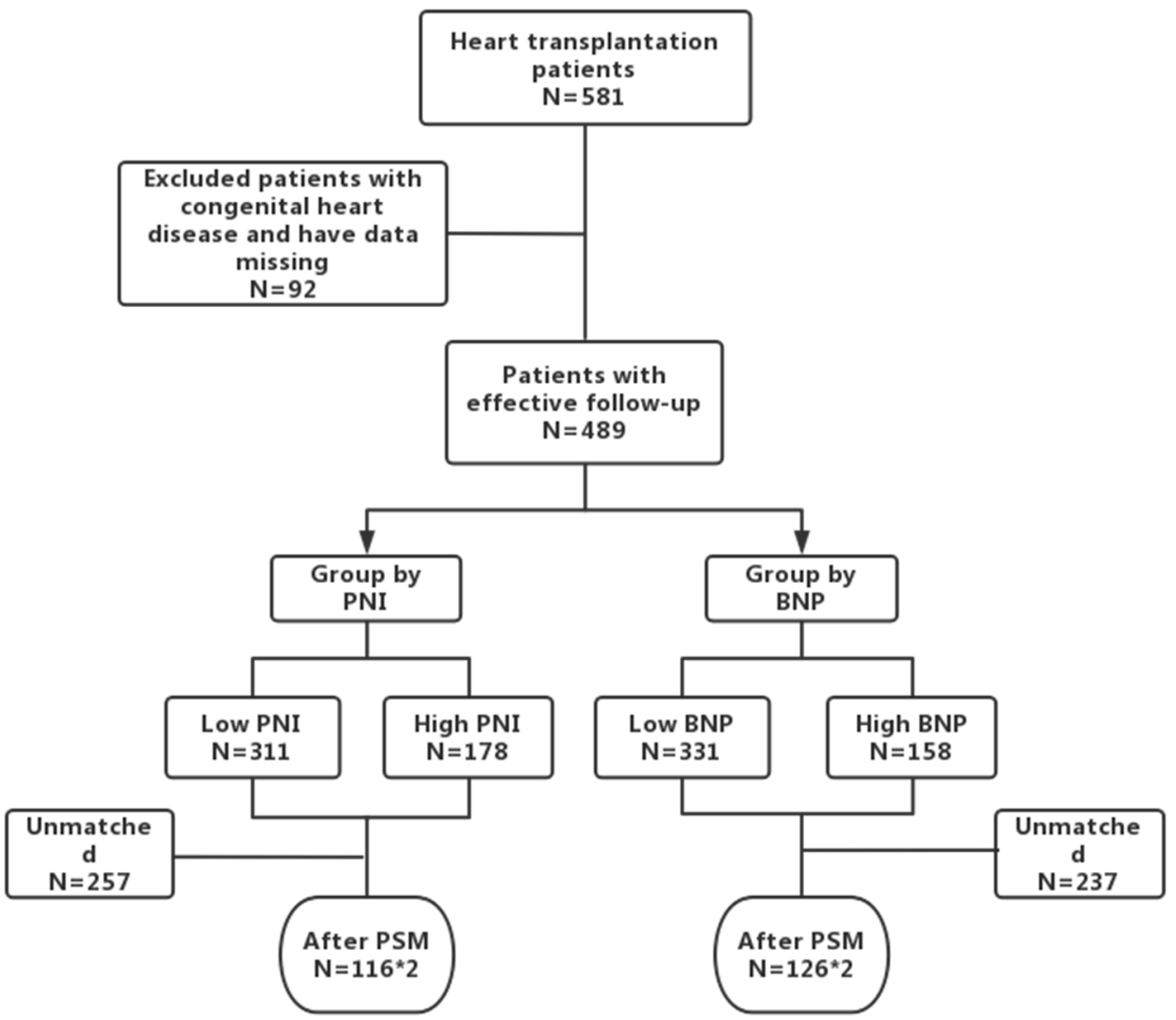

Figure 1. Study cohort. ${ }^{*} 2$ means there were some pairs of patients after PSM.

Table 1. Baseline patient characteristics based on PNI and BNP.

\begin{tabular}{|c|c|c|c|c|c|c|c|}
\hline \multirow{2}{*}{ Variables } & \multirow{2}{*}{ Case $(n=489)$} & \multicolumn{2}{|c|}{ PNI } & \multicolumn{4}{|c|}{ BNP } \\
\hline & & Low $(n=311)$ & High $(n=178)$ & $p$-Value & Low $(n=331)$ & High $(n=158)$ & $p$-Value \\
\hline \multicolumn{8}{|l|}{ Demographic Index } \\
\hline Sex & & & & 0.747 & & & 0.519 \\
\hline Male & 383 & 245 & 138 & & 262 & 121 & \\
\hline Female & 106 & 66 & 40 & & 69 & 37 & \\
\hline Age & $47.57 \pm 12.65$ & $48.77 \pm 11.97$ & $45.47 \pm 13.52$ & 0.005 & $46.70 \pm 12.68$ & $49.38 \pm 12.42$ & 0.029 \\
\hline diagnosis & & & & 0.058 & & & 0.137 \\
\hline $\begin{array}{c}\text { Ischemic } \\
\text { cardiomyopathy }\end{array}$ & 95 & 64 & 31 & & 70 & 25 & \\
\hline $\begin{array}{l}\text { Non-ischemic } \\
\text { cardiomyopathy }\end{array}$ & 308 & 190 & 118 & & 197 & 111 & \\
\hline $\begin{array}{l}\text { Congenital heart } \\
\text { disease }\end{array}$ & 19 & 8 & 11 & & 15 & 4 & \\
\hline $\begin{array}{l}\text { Other heart diseases } \\
\text { recipient blood-type }\end{array}$ & 67 & 49 & 18 & 0.707 & 49 & 18 & 0.023 \\
\hline
\end{tabular}


Table 1. Cont.

\begin{tabular}{|c|c|c|c|c|c|c|c|}
\hline \multirow{2}{*}{ Variables } & \multirow{2}{*}{ Case $(n=489)$} & \multicolumn{2}{|c|}{ PNI } & \multicolumn{4}{|c|}{ BNP } \\
\hline & & Low $(n=311)$ & High $(n=178)$ & $p$-Value & Low $(n=331)$ & High $(n=158)$ & $p$-Value \\
\hline $\mathrm{A}$ & 164 & 109 & 55 & & 111 & 53 & \\
\hline B & 134 & 86 & 48 & & 78 & 56 & \\
\hline $\mathrm{AB}$ & 31 & 18 & 13 & & 22 & 9 & \\
\hline $\mathrm{O}$ & 160 & 98 & 62 & & 120 & 40 & \\
\hline recipient BMI & $23.00 \pm 7.46$ & $22.95 \pm 8.74$ & $23.07 \pm 4.42$ & 0.874 & $23.54 \pm 8.63$ & $21.87 \pm 3.80$ & 0.020 \\
\hline recipient/donor BMI & $1.04 \pm 0.26$ & $1.05 \pm 0.26$ & $1.02 \pm 0.28$ & 0.131 & $1.02 \pm 0.27$ & $1.08 \pm 0.25$ & 0.016 \\
\hline recipient/donor age & $0.81 \pm 0.40$ & $0.78 \pm 0.35$ & $0.87 \pm 0.46$ & 0.012 & $0.83 \pm 0.41$ & $0.78 \pm 0.36$ & 0.17 \\
\hline recipient/donor sex & & & & 0.951 & & & 0.703 \\
\hline Male/Female & 35 & 22 & 13 & & 25 & 10 & \\
\hline Male/Male & 347 & 222 & 125 & & 237 & 110 & \\
\hline Female/Male & 78 & 50 & 28 & & 52 & 26 & \\
\hline Female/Female & 29 & 17 & 12 & & 17 & 12 & \\
\hline \multicolumn{2}{|c|}{ recipient/donor blood-type } & & & 0.627 & & & 0.133 \\
\hline identical & 400 & 252 & 148 & & 277 & 123 & \\
\hline different & 89 & 59 & 30 & & 54 & 35 & \\
\hline \multirow[t]{2}{*}{$\begin{array}{l}\text { Heart surgery history } \\
\text { (Yes) }\end{array}$} & 132 & 89 & 43 & 0.286 & 90 & 42 & 0.888 \\
\hline & & 190 & 99 & & & & \\
\hline \multicolumn{2}{|c|}{ Charlson Comorbidity Index } & & & 0.582 & & & 0.510 \\
\hline 1 & 134 & 84 & 50 & & 87 & 47 & \\
\hline 2 & 60 & 40 & 20 & & 44 & 16 & \\
\hline$\geq 3$ & 16 & 12 & 4 & & 11 & 5 & \\
\hline waiting time & & $29.64 \pm 15.56$ & $29.31 \pm 11.51$ & $<0.001$ & $29.98 \pm 14.03$ & $28.56 \pm 14.57$ & 0.955 \\
\hline \multicolumn{8}{|l|}{ Preoperative Therapy } \\
\hline preoperative IABP & 8 & 6 & 2 & 0.5 & 1 & 7 & 0.001 \\
\hline preoperative ECMO & 6 & 4 & 2 & 0.875 & 2 & 4 & 0.070 \\
\hline preoperative ARB & 85 & 54 & 31 & 0.988 & 58 & 27 & 0.906 \\
\hline preoperative ACEI & 163 & 86 & 77 & $<0.001$ & 123 & 40 & 0.009 \\
\hline $\begin{array}{l}\text { preoperative } \\
\text { dopamine }\end{array}$ & 291 & 198 & 93 & 0.013 & 180 & 111 & 0.001 \\
\hline preoperative BB & 381 & 233 & 148 & 0.035 & 264 & 117 & 0.155 \\
\hline \multicolumn{8}{|c|}{ Preoperative Blood Index } \\
\hline $\mathrm{Hb}$ & $133.15 \pm 23.34$ & $128.71 \pm 23.57$ & $140 / 90 \pm 20.51$ & $<0.001$ & $134.37 \pm 24.70$ & $130.56 \pm 19.67$ & 0.093 \\
\hline ALT & $71.19 \pm 295.91$ & $74.32 \pm 249.15$ & $65.72 \pm 364.21$ & 0.758 & $66.58 \pm 289.29$ & $80.85 \pm 310.03$ & 0.618 \\
\hline AST & $60.25 \pm 253.23$ & $62.76 \pm 248.85$ & $55.88 \pm 261.37$ & 0.773 & $53.43 \pm 211.17$ & $74.54 \pm 324.03$ & 0.389 \\
\hline D-dimer & $6.77 \pm 7.81$ & $6.18 \pm 7.70$ & $7.79 \pm 7.93$ & 0.029 & $6.42 \pm 7.92$ & $7.49 \pm 7.55$ & 0.161 \\
\hline troponin & $\begin{array}{c}1034.23 \pm \\
5713.50\end{array}$ & $\begin{array}{c}1029.75 \pm \\
5154.90\end{array}$ & $\begin{array}{c}1042.06 \pm \\
6592.53\end{array}$ & 0.982 & $\begin{array}{c}1135.31 \pm \\
6313.58\end{array}$ & $822.47 \pm 4197.02$ & 0.572 \\
\hline $\mathrm{Cr}$ & $99.24 \pm 52.38$ & $103.37 \pm 60.19$ & $92.04 \pm 33.70$ & 0.021 & $95.56 \pm 47.65$ & $106.96 \pm 60.55$ & 0.024 \\
\hline RBC & $4.50 \pm 1.53$ & $4.37 \pm 1.82$ & $4.73 \pm 0.78$ & 0.013 & $4.51 \pm 0.79$ & $4.49 \pm 2.45$ & 0.916 \\
\hline PLT & $180.45 \pm 66.77$ & $174.21 \pm 70.21$ & $191.35 \pm 58.91$ & 0.006 & $182.86 \pm 65.63$ & $175.39 \pm 69.05$ & 0.248 \\
\hline WBC & $6.79 \pm 4.65$ & $6.38 \pm 2.84$ & $7.51 \pm 6.69$ & 0.010 & $6.93 \pm 5.29$ & $6.51 \pm 2.89$ & 0.355 \\
\hline triglyceride (TG) & $1.12 \pm 0.69$ & $0.97 \pm 0.47$ & $1.39 \pm 0.90$ & $<0.001$ & $1.19 \pm 0.75$ & $0.98 \pm 0.52$ & 0.002 \\
\hline LDL & $2.08 \pm 0.93$ & $2.03 \pm 0.92$ & $2.16 \pm 0.94$ & 0.153 & $2.12 \pm 0.94$ & $2.00 \pm 0.89$ & 0.182 \\
\hline
\end{tabular}

\subsection{The Optimal Cut-Off Values of PNI and BNP for Estimating Prognosis}

In our study, we tried to obtain the optimal cut-off values of three potential indicators to predict survival with a ROC analysis method (Figure 2). During the process, the areas under the curve (AUC) of survival were $0.588(p<0.05,95 \%$ CI $0.333-0.555)$ for PNI, and $0.572(p<0.05,95 \%$ CI $0.423-0.776)$ for BNP. According to Youden index, the optimal cut-off values were verified as 49.345 for PNI, and 4397.500 for BNP.
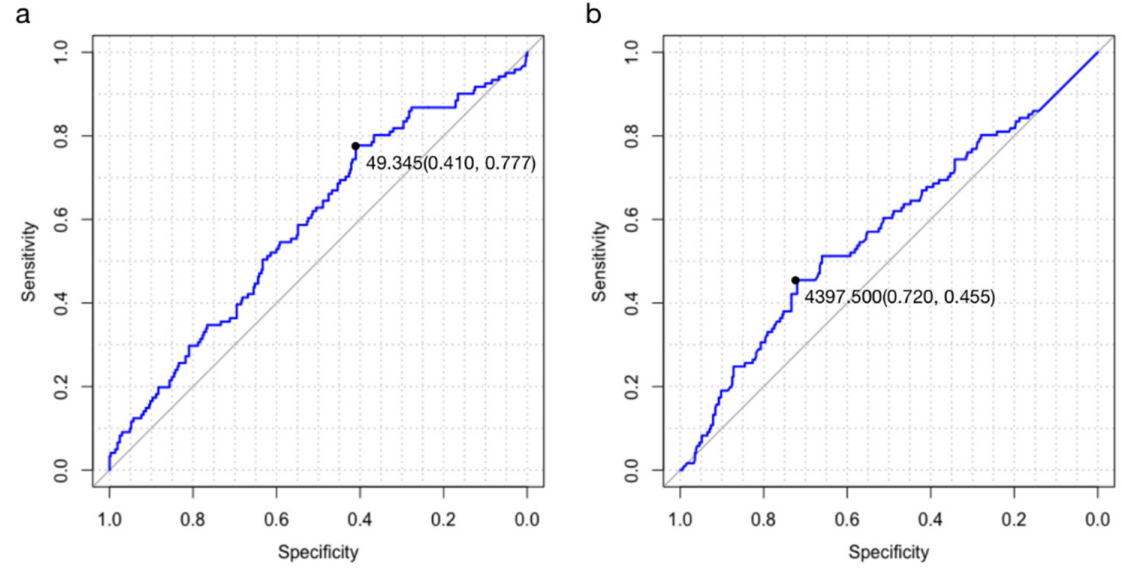

Figure 2. ROC curve for cut-off: (a) The ROC of PNI. (b) The ROC of BNP. 


\subsection{Baseline Characteristic of Different Groups}

Baseline clinical characteristics were shown and compared between two groups of each blood index separately (Table 1 ). Higher PNI was significantly related with younger age $(p=0.005)$, larger recipient/donor age ratio $(p=0.012)$, shorter waiting time $(p<0.001)$, more preoperative ACEI use $(p<0.001)$, less preoperative dopamine $(p=0.013)$, more preoperative BB use $(p=0.035)$, lower Cr level $(p=0.021)$, higher D-dimer, Hb, RBC, PLT, WBC, and triglyceride ( $p=0.029, p<0.001, p=0.013, p=0.006, p=0.01$, and $p<0.001)$.

In addition, lower BNP was significantly associated with younger age $(p=0.029)$, higher recipient BMI $(p=0.02)$, lower recipient/donor BMI $(p<0.016)$, less preoperative IABP use $(p=0.001)$, more preoperative ACEI use $(p=0.009)$, less preoperative dopamine use $(p=0.001)$, lower $\mathrm{Cr}$ level $(p=0.024)$, and higher triglyceride level $(p=0.002)$. There was no significant difference in other variables between two groups.

\subsection{Univariate and Multivariate Cox Analysis of OS of Patients with HTx}

The results presented the relationships between blood biomarkers and OS. The low PNI group had a more significant OS than that of high PNI group $(p<0.001$, Figure 3a), whereas the high BNP group was observed with worse OS than that of low the BNP group $(p<0.001$, Figure 3b). Multivariate analysis showed that older age (HR 1.025, 95\%CI, 1.009-1.042, $p=0.002$ ), low PNI level (HR 0613, 95\%CI 0.378-0.993, $p=0.047$ ), high BNP level (HR 1.542, 95\%CI 1.057-2.248, $p=0.024$ ), and long waiting time (HR 1.014, 95\%CI, $1.003-1.026, p=0.012$ ) were the independent protective factors for the prognostic of heart transplantation (Table 2).

a os

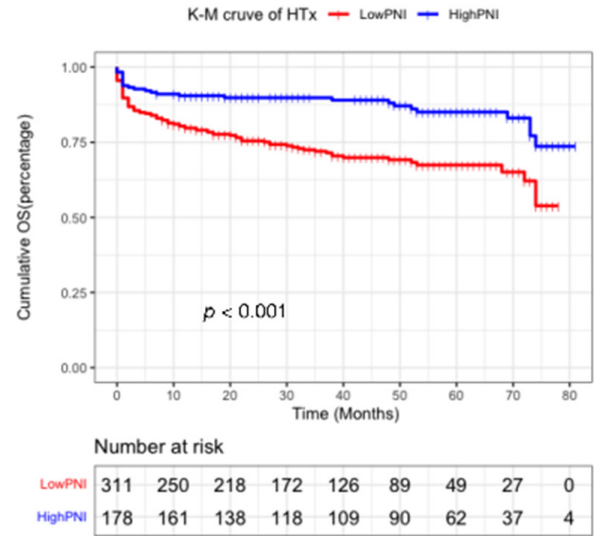

C os

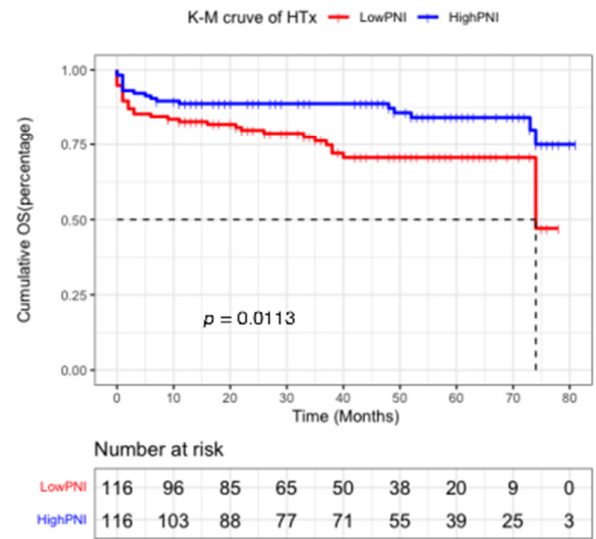

b os
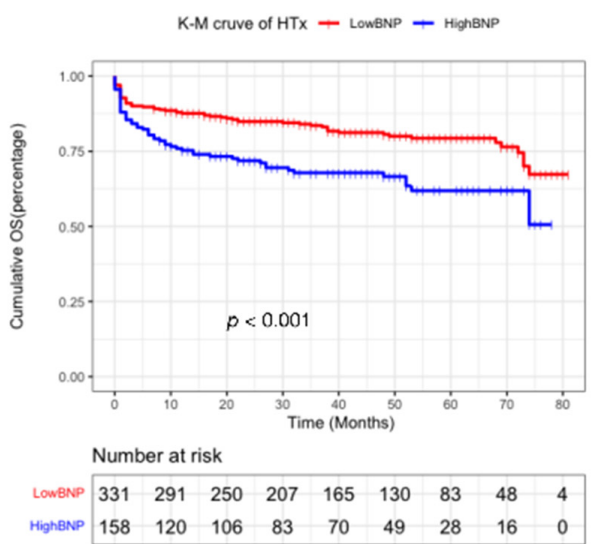

d os

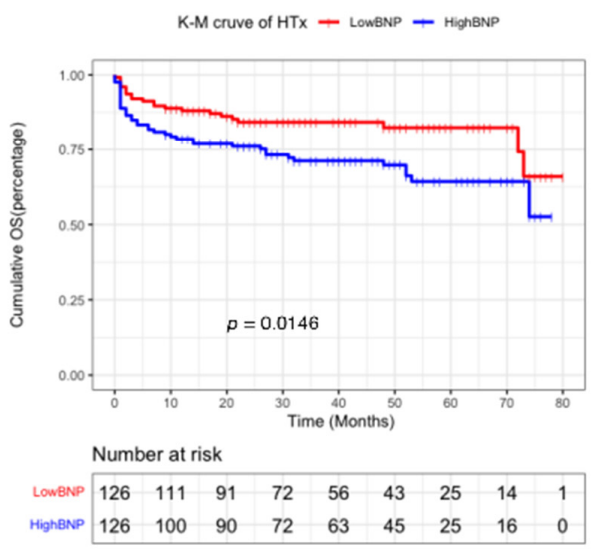

Figure 3. KM curve before and after PSM: (a) KM curve of PNI before PSM. (b) KM curve of PNI after PSM. (c) KM curve of BNP before PSM. (d) KM curve of BNP after PSM. 
Table 2. Univariate and multivariate analysis of overall survival in patients with heart transplantation.

\begin{tabular}{|c|c|c|c|c|c|c|}
\hline \multirow{2}{*}{ Variables } & \multicolumn{3}{|c|}{ Univariate Analysis } & \multicolumn{3}{|c|}{ Multivariate Analysis } \\
\hline & HR & $95 \% \mathrm{CI}$ & $p$ Value & HR & $95 \% \mathrm{CI}$ & $p$ Value \\
\hline \multicolumn{7}{|l|}{ Demographic index } \\
\hline Sex & 1.501 & $1.010-2.232$ & 0.045 & 1.243 & $0.807-1.913$ & 0.324 \\
\hline Age & 1.027 & $1.011-1.043$ & 0.001 & 1.025 & $1.009-1.042$ & 0.002 \\
\hline Diagnosis & 1.064 & $0.910-1.243$ & 0.437 & & & \\
\hline Recipient blood-type & 1.106 & $0.920-1.331$ & 0.283 & & & \\
\hline Recipient BMI & 1.014 & $0.989-1.040$ & 0.287 & & & \\
\hline Recipient/donor BMI & 1.372 & $0.695-2.707$ & 0.362 & & & \\
\hline Recipient/donor age & 0.662 & $0.401-1.093$ & 0.107 & & & \\
\hline Recipient/donor sex & 0.851 & $0.659-1.098$ & 0.215 & & & \\
\hline Recipient/donor blood-type & 1.226 & $1.013-1.485$ & 0.037 & 1.118 & $0.936-1.337$ & 0.220 \\
\hline Cardiac surgery history (Yes) & 1.308 & $0.890-1.921$ & 0.172 & & & \\
\hline Charlson Comorbidity Index & 1.124 & $0.944-1.338$ & 0.19 & & & \\
\hline Waiting time & 1.013 & $1.001-1.024$ & 0.036 & 1.014 & $1.003-1.026$ & 0.012 \\
\hline \multicolumn{7}{|l|}{ Preoperative therapy } \\
\hline Preoperative IABP & 3.374 & $1.239-9.187$ & 0.017 & 2.185 & $0.781-6.113$ & 0.136 \\
\hline Preoperative ECMO & 1.014 & $0.141-7.274$ & 0.989 & & & \\
\hline Preoperative ARB & 1.368 & $0.866-2.160$ & 0.179 & & & \\
\hline Preoperative ACEI & 0.506 & $0.331-0.773$ & 0.002 & 0.675 & $0.431-1.059$ & 0.087 \\
\hline Preoperative dopamine & 1.553 & $1.063-2.270$ & 0.023 & 1.339 & $0.901-1.988$ & 0.148 \\
\hline Preoperative BB & 0.704 & $0.475-1.044$ & 0.081 & & & \\
\hline \multicolumn{7}{|c|}{ Preoperative Blood index } \\
\hline $\mathrm{Hb}$ & 0.987 & $0.979-0.995$ & 0.002 & 0.997 & $0.987-1.007$ & 0.521 \\
\hline ALT & 1.000 & $0.999-1.001$ & 0.884 & & & \\
\hline AST & 1.000 & $1.000-1.001$ & 0.433 & & & \\
\hline D-dimer & 1.017 & $0.995-1.041$ & 0.137 & & & \\
\hline Troponin & 1.000 & $1.000-1.000$ & 0.668 & & & \\
\hline $\mathrm{Cr}$ & 1.002 & $1.000-1.005$ & 0.082 & & & \\
\hline $\mathrm{RBC}$ & 0.774 & $0.611-0.982$ & 0.035 & 0.993 & 0.881-1.119 & 0.913 \\
\hline PLT & 0.998 & $0.995-1.001$ & 0.235 & & & \\
\hline WBC & 1.014 & 0.990-1.039 & 0.242 & & & \\
\hline Triglyceride (TG) & 0.734 & $0.544-0.990$ & 0.043 & 0.932 & $0.670-1.296$ & 0.675 \\
\hline LDL & 1.111 & $0.910-1.357$ & 0.3 & & & \\
\hline PNI & 0.416 & $0.271-0.642$ & $<0.001$ & 0.613 & $0.378-0.993$ & 0.047 \\
\hline $\mathrm{BNP}$ & 1.917 & $1.340-2.743$ & $<0.001$ & 1.542 & $1.057-2.248$ & 0.024 \\
\hline
\end{tabular}

\subsection{Survival Analysis of HTx Patients of Different Level of PNI and BNP after PSM}

In the PNI cohort, $47.4 \%(232 / 489)$ of patients were successfully matched into pairs, as were $51.5 \%(252 / 489)$ of patients in the BNP cohort. The distribution of propensity score is presented in the Supplementary Materials. The density of propensity score for each arm was shown before and after matching, and those matched represented a balanced and heterogeneous distribution. Unmatched low PNI group and high BNP group had lower scores, whereas unmatched high PNI group and low BNP group had higher scores.

After matching, all baseline characteristics had the differences eliminated, and those variables were equally contributed between both two groups without affecting others for each cohort (Table 3). Kaplan-Meier analysis showed that patients with lower PNI, higher BNP had significantly worse overall survival rates (Figure 3c,d).

\subsection{Effectiveness Evaluation of BNP and PNI Level in Predicting the OS of HTx}

Next, we attempted to evaluate the effect of predictive potency blood biomarkers on survival. C-index test was used to analyze the data after matching the sensitivity, specificity, AUC, and concordance index of PNI, which were $77.7 \%, 41 \%, 0.594$, and 0.593 (0.554-0.634), respectively. As for BNP, the results respectively were $72 \%, 45.5 \%, 0.587,0.582$ (0.536-0.628) (Table 4). The ROC curve for PNI, BNP, and the combined indicator showed that PNI had a superior AUC value than BNP (0.584 and 0.587). Meanwhile, the potency 
of the combined prediction of PNI and BNP was higher than that of single prediction (AUC $=0.634$, c-index $=0.632,95 \%$ CI: 0.585-0.680) (Figure 4). The effects of PNI and BNP at peri-transplant period, 1 year, and 5 year were shown in the Supplementary Materials. As shown in Figure 5, KM analysis with lowest combined index had the best overall survival than other subgroup, the 1-year survival rate of each subgroup of combined index was $92.0 \%, 84.1 \%$, and $73.7 \%$ from low to high. All pairwise comparisons were significant at $p<0.05$.

Table 3. Propensity score matching analysis of patients with heart transplantation based on PNI and BNP.

\begin{tabular}{|c|c|c|c|c|c|c|}
\hline \multirow{2}{*}{ Variables } & \multicolumn{3}{|c|}{ PNI (after PSM) $(n=232)$} & \multicolumn{3}{|c|}{ BNP (after PSM) $(n=252)$} \\
\hline & Low $(n=116)$ & High $(n=116)$ & $p$ Value & Low $(n=126)$ & High $(n=126)$ & $p$ Value \\
\hline \multicolumn{7}{|l|}{ Demographic index } \\
\hline Sex & & & 0.872 & & & 0.479 \\
\hline Male & 91 & 92 & & 94 & 99 & \\
\hline Female & 25 & 24 & & 32 & 27 & \\
\hline Age & $45.99 \pm 12.29$ & $46.18 \pm 13.84$ & 0.912 & $47.50 \pm 12.35$ & $48.45 \pm 12.33$ & 0.541 \\
\hline Diagnosis & & & 0.913 & & & \\
\hline $\begin{array}{c}\text { Ischemic } \\
\text { cardiomyopathy }\end{array}$ & 20 & 20 & & 21 & 21 & \\
\hline $\begin{array}{l}\text { Non-ischemic } \\
\text { cardiomyopathy }\end{array}$ & 75 & 79 & & 85 & 85 & \\
\hline Congenital & 5 & 4 & & 4 & 4 & \\
\hline Other & 16 & 13 & & 16 & 16 & \\
\hline Recipient blood-type & & & 0.892 & & & \\
\hline A & 31 & 36 & & 48 & 38 & \\
\hline B & 36 & 32 & & 28 & 45 & \\
\hline $\mathrm{AB}$ & 9 & 9 & & 10 & 8 & \\
\hline $\mathrm{O}$ & 40 & 39 & & 40 & 35 & \\
\hline Recipient BMI & $23.04 \pm 4.24$ & $22.80 \pm 4.57$ & 0.667 & $22.41 \pm 4.66$ & $22.30 \pm 3.60$ & 0.843 \\
\hline Recipient/donor BMI & $1.04 \pm 0.26$ & $1.04 \pm 0.28$ & 0.890 & $1.06 \pm 027$ & $1.04 \pm 0.20$ & 0.453 \\
\hline Recipient/donor age & $0.89 \pm 0.40$ & $0.84 \pm 0.38$ & 0.363 & $0.79 \pm 0.37$ & $0.77 \pm 0.34$ & 0.646 \\
\hline Recipient/donor sex & & & 0.449 & & & 0.821 \\
\hline Male/Female & 4 & 9 & & 9 & 10 & \\
\hline Male/Male & 85 & 84 & & 86 & 88 & \\
\hline Female/Male & 23 & 18 & & 25 & 20 & \\
\hline Female/Female & 4 & 5 & & 6 & 8 & \\
\hline $\begin{array}{l}\text { Recipient/donor } \\
\text { blood-type }\end{array}$ & & & 0.714 & & & \\
\hline Identical & 92 & 95 & & 102 & 100 & \\
\hline Different & 24 & 21 & & 24 & 26 & \\
\hline $\begin{array}{c}\text { Heart surgery history } \\
\text { (Yes) }\end{array}$ & 36 & 30 & 0.383 & 26 & 28 & 0.759 \\
\hline $\begin{array}{l}\text { Charlson Comorbidity } \\
\text { Index }\end{array}$ & & & 0.902 & & & \\
\hline 0 & 72 & 68 & & 81 & 73 & \\
\hline 1 & 27 & 32 & & 27 & 37 & \\
\hline 2 & 14 & 13 & & 12 & 12 & \\
\hline$\geq 3$ & 3 & 3 & & 6 & 4 & \\
\hline Waiting time & $31.43 \pm 15.72$ & $29.27 \pm 11.79$ & 0.237 & $30.06 \pm 14.05$ & $28.75 \pm 14.60$ & 0.487 \\
\hline Preoperative therapy & & & & & & \\
\hline Preoperative IABP & 1 & 1 & 1.000 & 0 & 1 & 0.316 \\
\hline Preoperative ECMO & 2 & 1 & 0.561 & 1 & 0 & 0.316 \\
\hline Preoperative ARB & 16 & 20 & 0.468 & 24 & 21 & 0.622 \\
\hline Preoperative ACEI & 50 & 47 & 0.690 & 37 & 38 & 0.890 \\
\hline Preoperative dopamine & 63 & 64 & 0.895 & 83 & 82 & 0.762 \\
\hline Preoperative BB & 93 & 91 & 0.746 & 100 & 98 & 0.759 \\
\hline
\end{tabular}




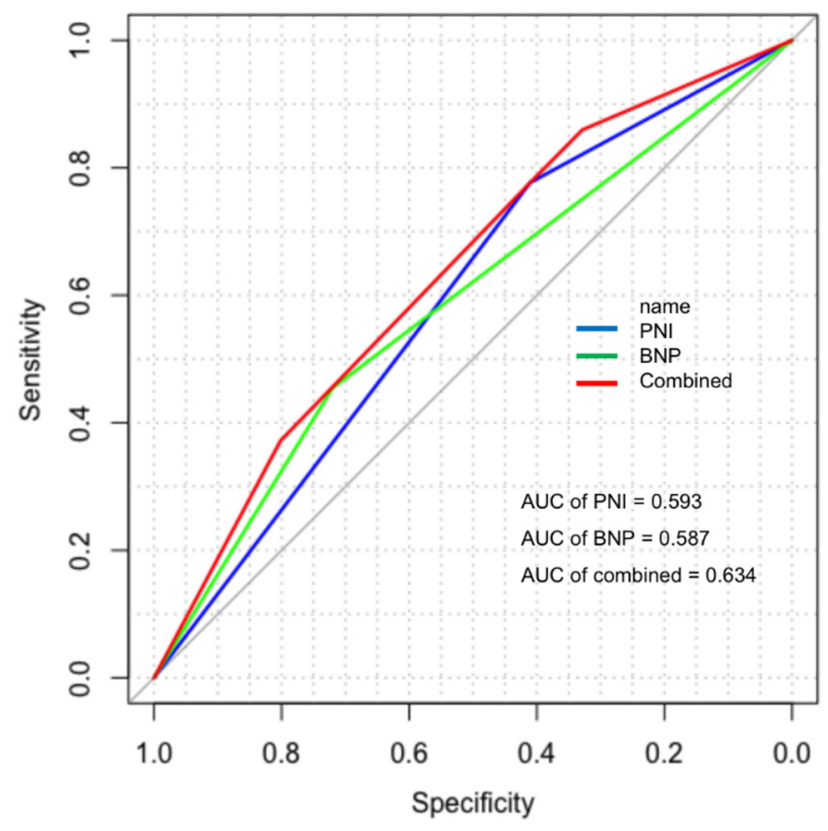

Figure 4. ROC curve for PNI, BNP, and combined indicator.

OS

K-M cruve of HTx + Low - Middle - High

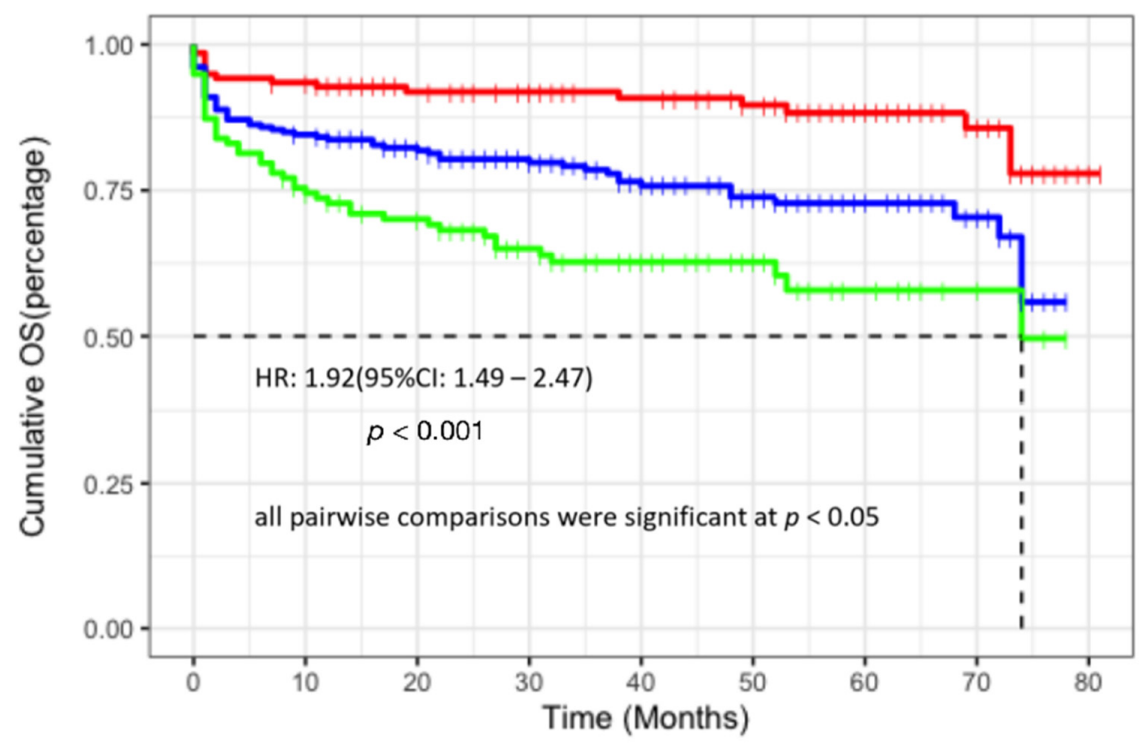

Number at risk

\begin{tabular}{r|ccccccccc|}
\cline { 2 - 10 } Low & 138 & 128 & 108 & 93 & 85 & 73 & 50 & 29 & 4 \\
Middle & 233 & 196 & 172 & 139 & 104 & 74 & 45 & 27 & 0 \\
High & 118 & 87 & 76 & 58 & 46 & 32 & 16 & 8 & 0 \\
\cline { 2 - 22 }
\end{tabular}

Figure 5. KM curve for combined indicators. 
Table 4. Diagnostic evaluation of PNI and BNP in OS of Patients with heart transplantation.

\begin{tabular}{cccc}
\hline Evaluation Index & PNI & BNP & Combined \\
\hline Sensitivity (\%) & 77.7 & 72.0 & 85.1 \\
Specificity $(\%)$ & 41.0 & 45.5 & 34.2 \\
AUC & 0.594 & 0.587 & 0.634 \\
C-index & $0.593(0.554-0.634)$ & $0.582(0.536-0.628)$ & $0.632(0.585-0.680)$ \\
\hline
\end{tabular}

\section{Discussion}

In the present study, we investigated whether nutritional status and preoperative heart failure were associated with OS in patients undergoing HTx. Since the clinical prognosis after HTx is not always predictable, it is important for the clinician to investigate risk factors and develop a prediction model. In this study, we aimed to explore the prognostic significance of immune-inflammatory indexes in patients underwent HTx. This study revealed that PNI and BNP were independent prognostic blood biomarkers for OS after HTx, especially in short-term survival. More significantly, combining BNP and PNI as a predicting tool for prognosis of HTx would be optimal in clinical practice.

PNI was first applied as an objective nutritional screening tool by Buzby et al. in 1980 [5], and it has been used as a nutritional biomarker to predict the prognosis in some cardiovascular diseases such as acute heart failure, coronary artery disease, and myocardial infarction [2-4]. However, there is no research to assess the role of PNI in HTx. Malnutrition is a physical condition comprising the reduction in calories, protein, and micro-nutrients and, consequently, leads to the weakness of immune defenses [6]. Patients waiting for HTx are always in end-stage HF status, and gastrointestinal congestion caused by HF leads to a loss of appetite [7]. Thus, it is not a rare condition in the patients waiting for HTx. Shirley et al. have applied three scoring systems to assess the nutrition status among outpatients with heart failure, including geriatric nutritional risk index (GNRI), controlling nutritional status (CONUT) score, and prognostic nutritional index (PNI) [8-10]. Moreover, the results suggested malnutrition was a common condition among outpatients with HF and had a significant relationship with increased mortality [11]. In the present study, our results demonstrated lower PNI level was associated with worse survival, which was consistent with previous research. Recipients with lower PNI level tended to be older and had a relatively higher rate of using preoperative assist devices and cardiac drugs, which would increase mortality and morbidity after HTx.

BNP is associated with cardiac dysfunction and worse hemodynamic parameters, and it is a common blood biomarker for diagnosis and prognosis in many cardiovascular diseases such as heart failure and coronary heart disease [12]. Left ventricular ejection fraction is widely used to assess the cardiac function, but Gardner et al. demonstrated nterminal-pro-BNP performed better in the prognostic prediction of HF than left ventricular ejection $[13,14]$. Moreover, there were some other studies focusing on the association between postoperative BNP level or donor BNP level and prognosis after HTx $[15,16]$. However, less attention was paid to investigate whether preoperative BNP level was able be the biomarker in the prognosis prediction. In the present study, univariable and multivariable analysis showed that BNP was an independent risk factor for overall survival $(p=0.024)$. Moreover, patients in higher BNP level groups had some characteristics such as older age, lower BMI, more preoperative IABP use, more preoperative ACEI use, and more preoperative dopamine use. Since age and waiting time on list have been proved to be independent risk factors in univariable and multivariable analysis, these factors would probably be responsible for worse survival in higher BNP level group. However, the present study implemented PSM to minimize confounding effects, and it would enhance the interpretability of the result that BNP is an independent indicator for OS after HTx.

Currently, there are some available score systems to assess the risk of cardiovascular surgery, such as EuroSCORE, EuroSCORE II, STS-SCORE, and so on [17-19]. Nevertheless, these scoring systems do not specially target the survival after heart transplantation. Thus, this study was conducted to investigate a simple risk prediction model that would be 
considered in clinical practice. In the present study, both PNI and BNP showed good diagnostic accuracy for the survival of HTx, especially in short-term survival, and the combined accuracy and validity of the two indicators were better than that of a single index. These findings extend and corroborate previous work in which evaluating PNI and $\mathrm{BNP}$ together was a potential clinical preoperative intervention target in forecasting the prognosis of patients underwent HTx.

\section{Conclusions}

Our study confirmed the clinical value of PNI and BNP as a screening tool in predicting the prognosis of heart transplantation since low PNI level and high BNP level are associated with poor survival. Moreover, the combined index showed better efficiency in predicting survival, especially short-term survival, and if it was poor, more targeted treatment should be applied to improve patients' physical conditions before transplantation.

Supplementary Materials: The following supporting information can be downloaded at: https: / / www.mdpi.com/article/10.3390/jcdd9020040/s1, Figure S1 PSM distribution for PNI and BNP. Table S1 Diagnostic Evaluation of PNI and BNP in peri-transplant period survival. Table S2 Diagnostic Evaluation of PNI and BNP in 1-year survival. Table S3 Diagnostic Evaluation of PNI and BNP in 5-year survival.

Author Contributions: F.L., S.C. and N.D. conceptualized and designed the study and critically reviewed and revised the manuscript. Z.C. and J.T. carried out the statistical analyses and drafted the initial manuscript. L.X., Y.L. and B.D. participated in performance of the research and critically reviewed and revised the manuscript. All authors approved the final manuscript as submitted and agreed to be accountable for all aspects of the work. All authors have read and agreed to the published version of the manuscript.

Funding: This study was supported by the National Natural Science Foundation of China (81974034).

Institutional Review Board Statement: Donation after brain death has become the unique source of graft for heart transplantation in China since 1 January 2015. All donor grafts were allocated by the China Organ Transplant Response System according to Chinese laws. The retrospective study protocol was approved by the Ethics Committee of Tongji Medical College of Huazhong University of Science and Technology (IORG NO: IORG0003571) and performed in accordance with the national program for deceased organ donation in China (national protocol for China category I). The clinical and research activities complied with the Declarations of Helsinki and Istanbul.

Informed Consent Statement: Informed consent was obtained from all subjects involved in the study.

Data Availability Statement: All data are included in this manuscript.

Acknowledgments: This research is attributed to the Department of Cardiovascular Surgery, Union Hospital, Tongji Medical College, Huazhong University of Science and Technology. We are grateful to Jing Zhang, Guohua Wang, and Xiusi Xiongti for their generous assistance.

Conflicts of Interest: The authors declare no conflict of interest.

\section{References}

1. McMurray, J.J.; Adamopoulos, S.; Anker, S.D.; Auricchio, A.; Bohm, M.; Dickstein, K.; Falk, V.; Filippatos, G.; Fonseca, C.; Gomez-Sanchez, M.A.; et al. ESC guidelines for the diagnosis and treatment of acute and chronic heart failure 2012: The Task Force for the Diagnosis and Treatment of Acute and Chronic Heart Failure 2012 of the European Society of Cardiology. Developed in collaboration with the Heart Failure Association (HFA) of the ESC. Eur. J. Heart Fail. 2012, 14, 803-869. [CrossRef] [PubMed]

2. Cheng, Y.L.; Sung, S.H.; Cheng, H.M.; Hsu, P.F.; Guo, C.Y.; Yu, W.C.; Chen, C.H. Prognostic Nutritional Index and the Risk of Mortality in Patients With Acute Heart Failure. J. Am. Heart Assoc. 2017, 6, e004876. [CrossRef] [PubMed]

3. Doi, S.; Iwata, H.; Wada, H.; Funamizu, T.; Shitara, J.; Endo, H.; Naito, R.; Konishi, H.; Tsuboi, S.; Ogita, M.; et al. A novel and simply calculated nutritional index serves as a useful prognostic indicator in patients with coronary artery disease. Int. J. Cardiol. 2018, 262, 92-98. [CrossRef] [PubMed]

4. Keskin, M.; Hayiroglu, M.I.; Keskin, T.; Kaya, A.; Tatlisu, M.A.; Altay, S.; Uzun, A.O.; Borklu, E.B.; Guvenc, T.S.; Avci, I.I.; et al. A novel and useful predictive indicator of prognosis in ST-segment elevation myocardial infarction, the prognostic nutritional index. Nutr. Metab. Cardiovasc. Dis. 2017, 27, 438-446. [CrossRef] [PubMed] 
5. Buzby, G.P.; Mullen, J.L.; Matthews, D.C.; Hobbs, C.L.; Rosato, E.F. Prognostic nutritional index in gastrointestinal surgery. Am. J. Surg. 1980, 139, 160-167. [CrossRef]

6. Rahman, A.; Jafry, S.; Jeejeebhoy, K.; Nagpal, A.D.; Pisani, B.; Agarwala, R. Malnutrition and Cachexia in Heart Failure. JPEN J. Parenter Enteral. Nutr. 2016, 40, 475-486. [CrossRef] [PubMed]

7. Okoshi, M.P.; Romeiro, F.G.; Paiva, S.A.; Okoshi, K. Heart failure-induced cachexia. Arq. Bras. Cardiol. 2013, 100, $476-482$. [CrossRef]

8. Nishi, I.; Seo, Y.; Hamada-Harimura, Y.; Yamamoto, M.; Ishizu, T.; Sugano, A.; Sato, K.; Sai, S.; Obara, K.; Suzuki, S.; et al. Geriatric nutritional risk index predicts all-cause deaths in heart failure with preserved ejection fraction. ESC Heart Fail. 2019, 6, 396-405. [CrossRef]

9. Hollander, S.A.; Schultz, L.M.; Dennis, K.; Hollander, A.M.; Rizzuto, S.; Murray, J.M.; Rosenthal, D.N.; Almond, C.S. Impact of ventricular assist device implantation on the nutritional status of children awaiting heart transplantation. Pediatr. Transplant. 2019, 23, e13351. [CrossRef] [PubMed]

10. Murphy, L.; Gray, A.; Joyce, E. Anabolism to Catabolism: Serologic Clues to Nutritional Status in Heart Failure. Curr. Heart Fail. Rep. 2019, 16, 189-200. [CrossRef] [PubMed]

11. Pureza, V.; Florea, V.G. Mechanisms for cachexia in heart failure. Curr. Heart Fail. Rep. 2013, 10, 307-314. [CrossRef] [PubMed]

12. Talha, S.; Charloux, A.; Piquard, F.; Geny, B. Brain natriuretic peptide and right heart dysfunction after heart transplantation. Clin. Transplant. 2017, 31, e12969. [CrossRef] [PubMed]

13. Wang, H.; Li, Y.Y.; Chai, K.; Zhang, W.; Li, X.L.; Dong, Y.G.; Zhou, J.M.; Huo, Y.; Yang, J.F. [Contemporary epidemiology and treatment of hospitalized heart failure patients in real clinical practice in China]. Zhonghua Xin Xue Guan Bing Za Zhi 2019, 47, 865-874. [CrossRef] [PubMed]

14. Gardner, R.S.; Ozalp, F.; Murday, A.J.; Robb, S.D.; McDonagh, T.A. N-terminal pro-brain natriuretic peptide. A new gold standard in predicting mortality in patients with advanced heart failure. Eur. Heart J. 2003, 24, 1735-1743. [CrossRef] [PubMed]

15. Vorlat, A.; De Hous, N.; Vervaecke, A.J.; Vermeulen, T.; Van Craenenbroeck, E.; Heidbuchel, H.; Rodrigus, I.; Van Donink, W.; Ancion, A.; Van Cleemput, J.; et al. Biomarkers and Donor Selection in Heart Transplantation. Transplant. Proc. 2019, 51, 1673-1678. [CrossRef] [PubMed]

16. Franekova, J.; Hoskova, L.; Secnik, P., Jr.; Pazdernik, M.; Kotrbata, M.; Kubicek, Z.; Jabor, A. The role of timely measurement of galectin-3, NT-proBNP, cystatin C and hsTnT in predicting prognosis and heart function after heart transplantation. Clin. Chem. Lab. Med. 2016, 54, 339-344. [CrossRef] [PubMed]

17. Nashef, S.A.; Roques, F.; Michel, P.; Gauducheau, E.; Lemeshow, S.; Salamon, R. European system for cardiac operative risk evaluation (EuroSCORE). Eur. J. Cardiothorac. Surg. 1999, 16, 9-13. [CrossRef]

18. Nashef, S.A.; Roques, F.; Sharples, L.D.; Nilsson, J.; Smith, C.; Goldstone, A.R.; Lockowandt, U. EuroSCORE II. Eur. J. Cardiothorac. Surg. 2012, 41, 734-744. [CrossRef]

19. Shahian, D.M.; O’Brien, S.M.; Filardo, G.; Ferraris, V.A.; Haan, C.K.; Rich, J.B.; Normand, S.L.; DeLong, E.R.; Shewan, C.M.; Dokholyan, R.S.; et al. The Society of Thoracic Surgeons 2008 cardiac surgery risk models: Part 1-Coronary artery bypass grafting surgery. Ann. Thorac. Surg. 2009, 88, S2-S22. [CrossRef] [PubMed] 